\section{Síndrome nefrótico e injuria renal aguda con microangiopatía trombótica en mujer con enfermedad celíaca. Caso clínico}

\author{
JORGE VEGA ${ }^{1,2,3}$, RIENZI DÍAZ ${ }^{2,4}$, \\ GONZALO P. MÉNDEZ ${ }^{5}$, HELMUTH GOECKE ${ }^{1,2,6}$
}

\section{Nephrotic syndrome and acute kidney injury associated with celiac disease. Report of one case}

Celiac disease may be associated with other autoimmune diseases and exceptionally with glomerulopathies and nephrotic syndrome. Associations have been reported with IgA nephropathy, membranoproliferative glomerulonephritis, membranous glomerulopathy and minimal change disease. We report a 63-year-old woman who simultaneously presented with massive nephrotic syndrome (proteinuria $46 \mathrm{~g} /$ day) and cachexia due to a malabsorption syndrome secondary to celiac disease. The course of her diseases was complicated with cardiomyopathy due to severe malnutrition, septic shock, acute kidney injury that required dialysis for seven weeks and severe hypertension. A renal biopsy showed a membranoproliferative pattern of injury secondary to a thrombotic microangiopathy and diffuse podocyte damage. Four years later, the patient was in good general health, the glomerular filtration rate was 30 $\mathrm{ml} / \mathrm{min} / 1.73 \mathrm{~m}^{2}$ and there was non-nephrotic proteinuria.

(Rev Med Chile 2013; 141: 381-387).

Key words: Acute kidney injury; Nephrotic syndrome; Shock, septic.

'Servicio de Medicina,
Sección de Nefrología,
Hospital Naval Almirante
Nef, Viña del Mar. Chile.
Escuela de Medicina,
Universidad de Valparaíso,
Valparaíso. Chile.
${ }^{3}$ Servicio de Medicina,
Sección Nefrología,
Hospital Dr. Gustavo Fricke,
Viña del Mar. Chile.
4Unidad de Cuidados
Intensivos, Clínica Reñaca,
Viña del Mar. Chile.
${ }^{5}$ Departamento de
Anatomía Patológica,
Facultad de Medicina,
Pontificia Universidad
Católica de Chile.
${ }^{6}$ Departamento de
Nefrología, Escuela de
Medicina, Pontificia
Universidad Católica de
Chile.
Recibido el 17 de mayo de
2012 , aceptado el 12 de
septiembre de 2012.
Correspondencia a:
Dr. Jorge Vega
5 Norte 1035,
Viña del Mar.
Fono: $56-32-2974237$
Fax: $56-32-2970050$.
E-mail: jvegastieb@gmail.
com

L a enfermedad celíaca (EC) es una enteropatía inmuno-mediada que puede asociarse a otras enfermedades autoinmunes como síndrome de Sjögren, diabetes mellitus insulinodependiente, trastornos tiroideos, dermatitis herpetiforme, lupus eritematoso, enfermedad de Addison, hepatitis, cirrosis biliar primaria, artritis reumatoidea, polimiositis, miastenia gravis, alopecía areata y síndrome antifosfolípidos ${ }^{1-5}$. Excepcionalmente la EC se ha asociado a glomerulonefritis (GN) o síndrome nefrótico (SN). Se han comunicado asociaciones con GN por IgA (GN-IgA), glomerulopatía membranosa, GN membranoproliferativa (GNMP) y enfermedad de cambios mínimos $(\mathrm{ECM})^{1-4,6-12}$.

Comunicamos el caso de una paciente que presentó simultáneamente manifestaciones clínicas de EC y SN, cuya biopsia renal (BR) mostró una GN con patrón membranoproliferativo secun- dario a una microangiopatía trombótica (MAT) crónica. La asociación de MAT de compromiso renal y EC no ha sido previamente descrita en la literatura.

\section{Caso clínico}

Mujer de 63 años con antecedentes de psoriasis e histerectomía por miomatosis. Dos de sus 3 hijos desarrollaron diabetes mellitus insulino dependiente a los 18 años de edad y otro espondilitis anquilosante. Desde hacía 2 meses presentaba diarrea, baja de peso y últimamente hematoquezia, por lo que fue hospitalizada. Al examen estaba muy enflaquecida y pálida, tenía uñas quebradizas, caída del cabello, lengua despapilada y edema de las extremidades inferiores. Existía anemia con microcitosis, hipocromía y macrocitosis; déficit 
de hierro y ácido fólico, VHS elevada, trombocitosis, hipoalbuminemia, proteinuria masiva (46,3 g/24 h) y disfunción renal (Tabla 1). No se observó hematuria, leucocituria ni cilindruria en el sedimento de orina. Clínicamente, se diagnosticó una EC y SN.

La panendoscopia mostró atrofia marcada de la mucosa duodenal y la biopsia alteraciones características de EC. Los anticuerpos antitransglutaminasa tisular $\mathrm{y}$ anti-endomisio fueron positivos, los antinucleares, anti-DNA, ANCA y factor reumatoideo negativos. Las concentraciones de IgG, IgA, IgM, C’3 y C'4 fueron normales. La electroforesis de proteínas plasmáticas no mostró un peak monoclonal y la inmunofijación en orina no demostró presencia de cadenas livianas. La serología para $\mathrm{VIH}$, virus B y C fue negativa. Se indicó vitamina B12, folatos, hierro, globos rojos y alimentación parenteral total (NPTC). El mielograma mostró hiperplasia de las 3 series. Se realizó escáner sin contraste que mostró un bazo muy pequeño ( $43 \times 20 \times 18 \mathrm{~mm}$ ), edema del tejido celular subcutáneo, ascitis y derrame pleural. Al quinto día del ingreso presentó una crisis convulsiva generalizada y se prescribió fenitoína. La resonancia magnética mostró defectos nodulares milimétricos en la sustancia blanca subcortical. Al séptimo día presentó edema pulmonar agudo y cayó en shock. Fue trasladada a UCI y conectada a ventilación mecánica, requiriendo dosis altas de noradrenalina y dopamina. Se administró hidrocortisona $300 \mathrm{mg} /$ día. Las enzimas cardiacas (CK-MB y troponinas) fueron persistentemente normales. El ecocardiograma mostró dilatación de aurícula izquierda, ventrículo izquierdo dilatado con hipoquinesia difusa y disfunción ventricular izquierda sistólica y diastólica con fracción de eyección 32\% (Simpson) e hipertensión pulmonar. Al día siguiente apareció fiebre elevada que hizo sospechar sepsis y cayó en anuria. Se indicó terapia antibiótica con tazobactam y ertapenem y se instaló diálisis peritoneal. En los hemocultivos se aisló Klebsiella pneumoniae que se presumió originada en los senos paranasales, que estaban velados y desde donde se cultivó el mismo germen. En los días siguientes permaneció en ventilación mecánica y diálisis peritoneal, recibiendo NPTC y catecolaminas. El día 15 se retiró la peritoneodiálisis y se inició hemofiltración veno-venosa continua (HFVVC). El ecocardiograma efectuado al día 17 del ingreso mostró notable mejoría de la función cardiaca con una fracción de eyección de $75 \%$. Después del décimo día de falla renal y a 19 días de su admisión comenzó a presentar hipertensión arterial severa (PS > $200 \mathrm{mmHg}, \mathrm{PD}$ $>130 \mathrm{mmHg}$ ) que motivó el empleo de nitroglicerina, captopril, amlodipino y bisoprolol para su buen control. Simultáneamente se produjo una rápida anemización y caída en recuento de plaquetas desde 850.000 a $110.000 \mathrm{~mm}^{3}$, requiriendo transfusiones de glóbulos rojos en forma repetida. Un test de Coombs fue negativo. El día 30 se retiró de ventilación mecánica y se efectuó un escáner abdominal que objetivó lesiones nodulares hepáticas que hicieron sospechar una infección por hongos. Se prescribió fluconazol. El día 34 presentó una hemorragia digestiva secundaria a una úlcera subcardial, que se esclerosó endoscópicamente. El día 45 se efectuó una BR quirúrgica que incluyó 24 glomérulos. Los hallazgos fueron compatibles con una glomerulopatía de patrón mesangioproliferativo, sin evidencias de depósito de complejos inmunes ni factores de complemento y con caracteres de una lesión secundaria a microangiopatía trombótica crónica con daño pedicelar podocitario difuso (Figuras 1 y $2 \mathrm{~A}-2 \mathrm{~B}$ ). También se hallaron signos de enfermedad tubular extensa. El día 49 se discontinuó la HFVVC e inició hemodiálisis convencional, la que se suspendió a los 52 días de iniciada por mejoría de la función renal $\left(36,7 \mathrm{ml} / \mathrm{min} / 1,73 \mathrm{~m}^{2}\right)$. Fue dada de alta el día 66 de hospitalización, recibiendo prednisona

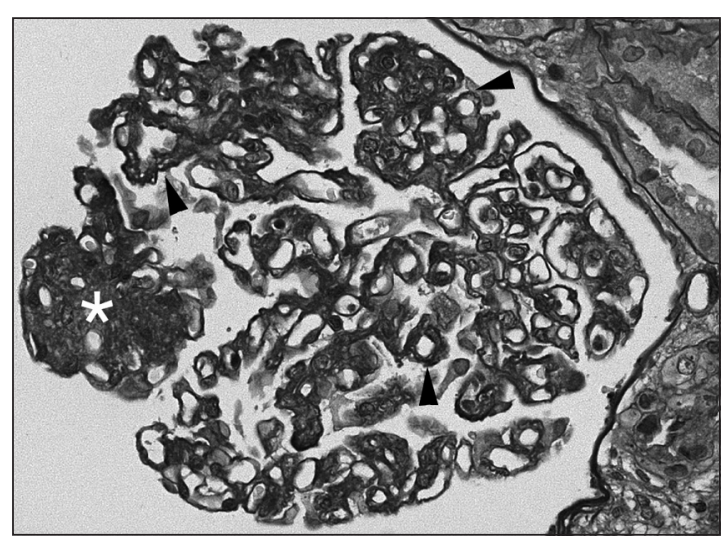

Figura 1. Microscopia óptica de un glomérulo con cambios membranoproliferativos caracterizados por múltiples zonas de delaminación y dobles contornos en sus asas capilares periféricas (cabezas de flecha) junto a segmentos de expansión mesangial por matriz y células (asterisco), que acentúa la lobulación del ovillo glomerular (tinción de PAS, 400x). 


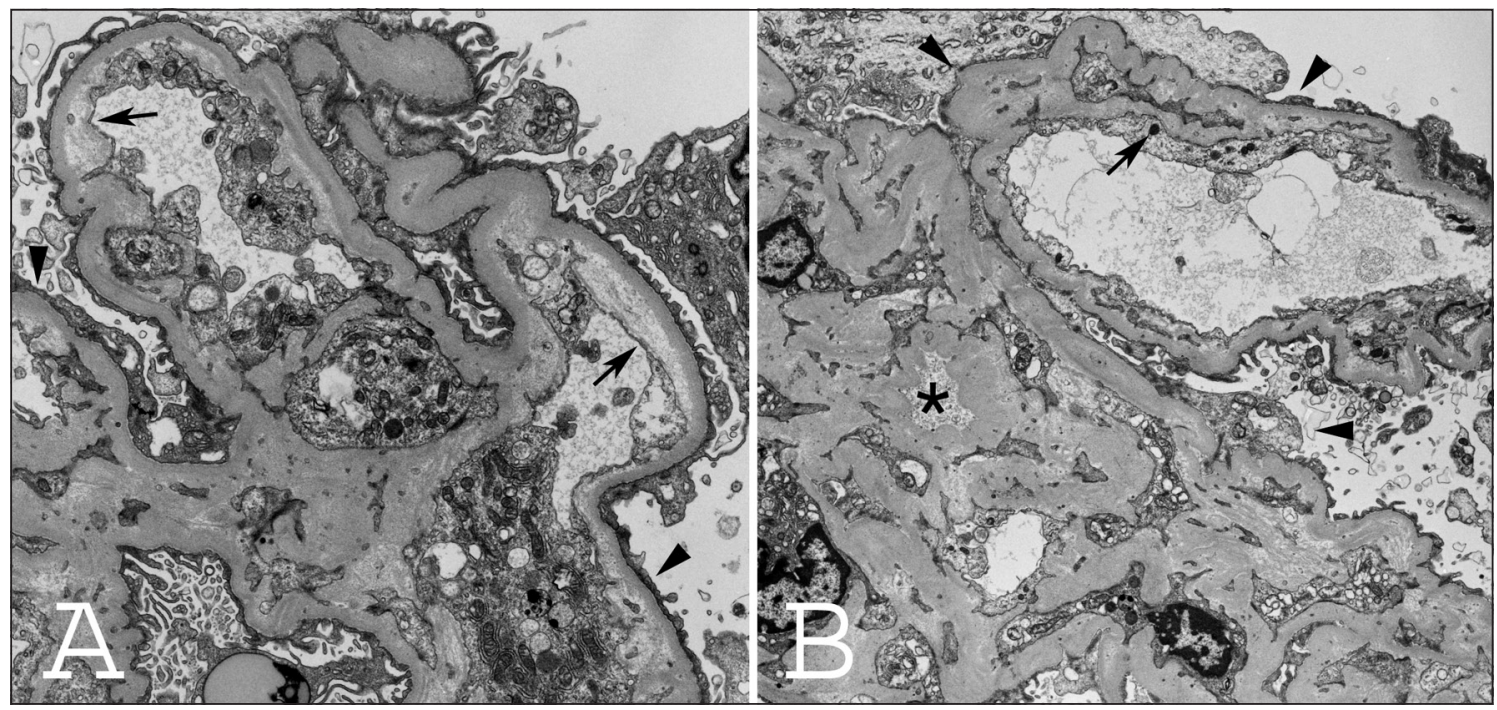

Figura 2. Microscopia electrónica. A. Zona del ovillo glomerular que presenta remodelación y daño endotelial temprano de los capilares glomerulares, caracterizado por expansión difusa de la lámina rara interna de la membrana basal por depósito de material electron-lúcido (flechas). No se observan depósitos densos que sugieran presencia de complejos inmunes (uranil acetato-citrato de plomo; 6000X). B. Segmento glomerular con cambios membranoproliferativos asociados a microangiopatía trombótica crónica. Se observa un doble contorno con interposición celular (flecha), en ausencia de depósito denso subendotelial. El mesangio está aumentado por acumulación de matriz (asterisco). (tetróxido de osmio-acetato de uranilo; 6000X). En ambas imágenes se reconoce borramiento pedicelar intenso (cabezas de flecha).

$30 \mathrm{mg} /$ día como terapia de su síndrome nefrótico y dieta sin gluten.

Durante los 7 meses posteriores al alta por persistencia del SN, estando en tratamiento con prednisona, enalapril, losartán, atorvastatina y furosemide; se indicó ciclofosfamida oral $100 \mathrm{mg} /$ día. Esta droga se utilizó durante $1 \frac{112}{2}$ año asociada a prednisona $5 \mathrm{mg} /$ día, obteniéndose una remisión parcial del síndrome nefrótico (Tabla 1). En el último control, a los 46 meses post-alta, el estado general de la paciente era muy bueno, su IMC era $27 \mathrm{~kg} / \mathrm{m}^{2}$, no había edemas, la proteinuria de $24 \mathrm{~h} 1.441 \mathrm{mg}$, el clearance de creatinina $30 \mathrm{ml} /$ $\mathrm{min} / 1,73 \mathrm{~m}^{2}$ y el sedimento de orina normal.

\section{Discusión}

Si bien las comunicaciones de asociación entre EC y GN son escasas, un estudio epidemiológico sueco mostró que el diagnóstico de GN era más frecuente en los enfermos portadores de EC y el empleo de diálisis o trasplante renal también era mayor ${ }^{13}$.

La enfermedad renal más frecuentemente aso- ciada a la EC es la GN-IgA $3,4,14,15$. Algunos autores han postulado que existe un nexo entre ambas patologías, porque los pacientes con GN-IgA tienen un aumento en la prevalencia de EC, dermatitis herpetiforme, anticuerpos antigliadina y antiendomisio y aumento en la sensibilidad rectal a gluten. La hematuria y la proteinuria disminuyen después de adoptar una dieta libre de gluten. Lo anterior permite plantear que pueda existir un mecanismo fisiopatológico común ${ }^{3,8,10,11,14-20}$. Se ha encontrado un aumento de los linfocitos $\mathrm{T}$ intraepiteliales en el intestino en la EC y glomerulopatías primarias, sugiriendo que el quiebre en la tolerancia oral a antígenos alimentarios o bacterianos pudiera jugar un rol en las $\mathrm{GN}^{21}$.

En la BR de esta paciente se encontró un patrón morfológico de una GNMP secundaria a una MAT crónica con presencia de una lesión pedicelar intensa de tipo enfermedad de cambios mínimos y necrosis tubular aguda (NTA). La existencia de una lesión tipo ECM fue planteada porque la paciente presentó un SN con proteinuria masiva, con hallazgos al examen ultraestructural de borramiento pedicelar difuso. Esto último fue lo de mayor significancia si se considera que en general 
Tabla 1. Exámenes de laboratorio durante la evolución de la enfermedad

\begin{tabular}{|c|c|c|c|c|}
\hline & $\begin{array}{l}\text { Ingreso al } \\
\text { hospital }\end{array}$ & $\begin{array}{c}\text { A } 24 \text { días del } \\
\text { egreso }\end{array}$ & $\begin{array}{c}\text { A } 7 \text { meses del } \\
\text { egreso }\end{array}$ & $\begin{array}{c}\text { A } 2 \text { años del } \\
\text { egreso }\end{array}$ \\
\hline Hematocrito (\%)/hemoglobina (g/dl) & $22,6 / 5,8$ & & $33,2 / 10,6$ & $31 / 10,1$ \\
\hline Ferremia (ug/dl) (50-160) & 10 & & & \\
\hline Saturación transferrina (\%) (20-55) & 4 & & & \\
\hline Ferritina (ng/ml) (30-400) & 10,7 & & & \\
\hline Ácido fólico (ng/ml) (3,1-14,4) & $<3,5$ & & & \\
\hline Vitamina B12 (pg/ml) (165-1.059) & 259 & & & \\
\hline Leucocitos $\left(\mathrm{mm}^{3}\right)$ & 6.200 & & 14.800 & 5.500 \\
\hline Plaquetas $\left(\mathrm{mm}^{3}\right)$ & 931.000 & & 372.000 & 193.000 \\
\hline VHS (mm/hora) & 80 & & 82 & 28 \\
\hline Protrombina (\%) (70-100) & 100 & 124 & & \\
\hline TTPK (segundos) (20-35) & 36,2 & 23 & & \\
\hline Proteína C (\%) & & 120 & & \\
\hline Proteína S (\%) & & 75,3 & & \\
\hline Resistencia a proteína $C$ activada $(>1,57)$ & & 5,4 & & \\
\hline Antitrombina (\%) & & 78 & & \\
\hline Anticoagulante lúpico & & Negativo & & \\
\hline Anti B2 glicoproteína IgG (< 20) & & 1,3 & & \\
\hline Anti B2 glicoproteína IgM (< 10) & & 3,0 & & \\
\hline Anticardiolipina IgG (< 20 GPL) & & 1,5 & & \\
\hline Anticardiolipina IgM (< 15 MPL) & & 13,0 & & \\
\hline VDRL & & Negativo & & \\
\hline Homocisteína (mmol/L) $(4,45-21,6)$ & & 10,5 & & \\
\hline Colesterol (mg/dl) (< 200) & 244 & 331 & 457 & 186 \\
\hline Triglicéridos (mg/dl) $(<150)$ & 317 & & 564 & 133 \\
\hline Proteínas (g/dl) $(6,4-8,3)$ & 5,8 & 5,9 & 6,0 & 6,1 \\
\hline Albumina (mg/dl) $(3,5-5,0)$ & 2,5 & 3,3 & 3,25 & 3,86 \\
\hline SGOT (U/L) (5-34) & 31 & & & \\
\hline SGPT (U/L) (1-55) & 7 & & & \\
\hline GGTP (U/L) (9-36) & 9 & & & \\
\hline LDH (U/L) (135-225) & 203 & & & \\
\hline Fosfatasas alcalinas (U/L) (40-150) & 109 & & & \\
\hline Bilirrubina total $(\mathrm{mg} / \mathrm{dl})(0,2-1,2)$ & 0.13 & & & \\
\hline Creatinina (mg/dl) $(0,6-1,1)$ & 1,57 & 1,31 & 1,76 & 1,85 \\
\hline Clearance de creatinina $24 \mathrm{~h}\left(\mathrm{ml} / \mathrm{min} / 1,73 \mathrm{~m}^{2}\right)$ & & 53,6 & 40,6 & 37,7 \\
\hline Proteinuria 24 h (mg) (< 150) & 46.300 & 23.544 & 8.129 & 3.419 \\
\hline Uremia (mg/dl) (18-42) & 45 & & & \\
\hline Ucicemia (mg/dl) $(2,6-6,0)$ & 6,0 & & 7,9 & \\
\hline Calcemia (mg/dl) $(8,4-10,2)$ & 9,1 & & & \\
\hline Amilasas (U/L) (25-125) & 347 & & & \\
\hline Glicemia (mg/dl) (65-100) & 138 & & 92 & 84 \\
\hline TSH (uUl/ml) $(0,27-4,20)$ & 3,51 & & & \\
\hline T3 $(\mathrm{nmol} / \mathrm{L})(1,3-3,1)$ & 1,09 & & & \\
\hline T4 (ug/dl) $(5,2-14,1)$ & 6,46 & & & \\
\hline Proteína $C$ reactiva $(\mathrm{mg} / \mathrm{L})(0,1-8,2)$ & 3,3 & & & \\
\hline $\operatorname{lgG}(\mathrm{mg} / \mathrm{dl})(700-1500)$ & 913 & & & \\
\hline $\lg A(\mathrm{mg} / \mathrm{dl})(80-400)$ & 234 & & & \\
\hline $\operatorname{lgM}(\mathrm{mg} / \mathrm{dl})(40-260)$ & 51 & & & \\
\hline $\mathrm{C}^{\prime} 3$ (mg/dl) $(0,83-1,93)$ & 102 & & & \\
\hline $\mathrm{C}^{\prime} 4(\mathrm{mg} / \mathrm{dl})(0,15-0,57)$ & 38 & & & \\
\hline
\end{tabular}


las formas de MAT crónica presentan un grado de borramiento pedicelar que es parcelar, aunque de variable extensión ${ }^{22}$. La NTA secundaria a la sepsis y al síndrome de bajo débito cardiaco - producto de disfunción ventricular izquierda secundaria a miocardiopatía por déficit nutricional; explican la falla renal aguda anúrica prolongada. La MAT, que no fue sospechada clínicamente y se conoció sólo al recibir el resultado de la $\mathrm{BR}$, es de más difícil interpretación; esto porque no existen casos de MAT de compromiso renal comunicados en pacientes con EC. La literatura incluye escasos reportes de pacientes con EC que presentaron eventos asociados a trombosis e hipercoagulabilidad, pero afectando predominantemente la circulación venosa y órganos como corazón, cerebro e intestino delgado ${ }^{23}$.

La MAT es un término que en patología renal se usa para describir alteraciones glomerulares y vasculares tanto en sus formas limitadas al riñón como aquellas asociadas a patologías sistémicas, y que son secundarias a múltiples formas de daño endotelial agudo y crónico activo o cicatrizal ${ }^{24}$. Conceptualmente, una MAT aguda corresponde a la presencia de microtrombos fibrino-plaquetarios oclusivos endocapilares en glomérulos y arteriolas, con edema y desprendimiento de la célula endotelial de su membrana basal, obstrucción vascular parcial o completa y fragmentación de eritrocitos $^{24-26}$. Las formas crónicas de MAT son la manifestación morfológica tanto de una lesión glomerular "cicatrizal" de eventos agudos previos como de formas subclínicas de daño endotelial crónico persistente ${ }^{24}$. Se caracterizan por cambios remodelativos intensos en las asas capilares glomerulares y en el mesangio que llevan al desarrollo de un patrón membranoproliferativo de lesión glomerular ${ }^{22,24}$. Estas alteraciones incluyen acumulación de material electrón-lúcido y detritus en el espacio subendotelial; neoformación de una "segunda membrana basal"; interposición celular entre ambas membranas basales; desplazamiento secundario del endotelio de su membrana basal original y engrosamiento de la pared del capilar glomerular. El mesangio usualmente está expandido por infiltración mononuclear y una relativa mayor cantidad de matriz, a veces con fibrillas colágenas maduras y detritus.

El análisis morfológico de nuestra paciente, demostró alteraciones glomerulares compatibles con una forma remodelativa o crónica de MAT, que en su conjunto fueron categorizadas como una GNMP secundaria. Las lesiones encontradas, especialmente los dobles contornos glomerulares, están probablemente representando la curación de un daño endotelial ocurrido en el pasado reciente de la paciente, que no fue evidenciado en el tejido examinado por cuanto la BR se efectuó a las 5 semanas del inicio de la falla renal, cuando su enfermedad iba en franca recuperación.

La MAT comúnmente se asocia al síndrome hemolítico-urémico o al púrpura trombocitopénico trombótico. En el primero, la MAT ocurre fundamentalmente en el riñón y en el segundo, en el sistema nervioso central. A su vez, la MAT tiene numerosas asociaciones y factores precipitantes destacando: infecciones bacterianas, defectos en el metabolismo de la vitamina B12, drogas, neoplasias, desordenes genéticos o adquiridos del sistema del complemento, anormalidades en la enzima ADAMTS 13, embarazo, infección por VIH, trasplante de médula ósea, policitemia vera, anemia de células falciformes, irradiación, hipertensión maligna, rechazo de trasplante, disproteinemias, enfermedades colágeno-vasculares y síndrome antifosfolípido ${ }^{22-26}$. En los pacientes con EC se ha encontrado más frecuentemente la existencia de anticuerpos antifosfolípidos que en los controles ${ }^{5,27}$.

La etiología de la MAT no tiene una explicación clara en nuestra paciente. Si bien presentó un shock séptico secundario a una infección por Klebsiella pneumoniae, la MAT se ha descrito asociada a infecciones causadas por otros gérmenes como E. coli O157:H7, Shigella, Campylobactery Streptococcus pneumoniae. Tampoco existían alteraciones en los niveles de vitamina B12, uso de drogas pro-trombóticas, embarazo, neoplasias, infección por VIH, trombofilia, enfermedades colágenovasculares, ni otros de los factores mencionados. Pensamos que la MAT en nuestra paciente pudo haber ocurrido por un defecto transitorio del sistema del complemento (factor $\mathrm{H}$ o I) o una alteración de la enzima ADAMTS 13, pero estos defectos no fueron estudiados y la biopsia renal no demostró depósito significativo de C’3 glomerular. La negatividad de C 3 en glomérulos en el examen de inmunofluorescencia no descarta del todo un defecto transitorio del complemento, por cuanto no se encontraron elementos de actividad aguda de microangiopatía trombótica al momento de la biopsia. Otra posibilidad es que haya existido 
un déficit del factor de crecimiento del endotelio vascular (VEGF-A), elemento fundamental para el funcionamiento normal del endotelio ${ }^{28-30}$. Esta proteína es producida por los podocitos glomerulares, los cuales estaban severamente dañados en esta paciente. De hecho, se ha comunicado que el uso de anticuerpos monoclonales contra VEGF-A (bevacizumab) en el tratamiento del cáncer en humanos, puede producir proteinuria, hipertensión, insuficiencia renal aguda y $\mathrm{MAT}^{28}$. En el modelo animal, se ha demostrado que la deficiencia de VEGF-A produce síndrome nefrótico e insuficiencia renal ${ }^{29}$. Por último, la presencia de hipertensión arterial severa de difícil manejo durante la falla renal aguda, podría ser explicada por la MAT puesto que no es una manifestación observada en la NTA clásica. La anemización y caída de las plaquetas simultáneas apoyan esta hipótesis.

En síntesis, comunicamos el caso de una asociación no descrita en la literatura de enfermedad celíaca y microangiopatía trombótica, en una paciente adulta que debutó con un síndrome nefrótico severo, complicándose con injuria renal aguda, sepsis, hipertensión arterial de difícil manejo y miocardiopatía severa por déficit nutricional; que evolucionó satisfactoriamente con el tratamiento instituido, observándose regresión de la miocardiopatía y recuperación parcial de la función renal y del SN.

\section{Referencias}

1. Prasad D, Khara HS, Gupta M, Sterman P. Celiac disease associated membranous nephropathy-a rare cause or coincidence? A case report. Cases J 2009; 2: 7018.

2. Soro S, Sánchez de la Nieta MD, Rivera F. Coeliac disease and membranous nephropathy. Nefrología 2009; 29: 479-81.

3. Jhaveri KD, D'Agati VD, Pursell R, Serur D. Coeliac sprue-associated membranoproliferative glomerulonephritis (MPGN). Nephrol Dial Transplant 2009; 24: 3545-8.

4. La Villa G, Pantaleo P, Tarquini R, Cirami L, Perfetto F, Manvcuso F, et al. Multiple immune disorders in unrecognized celiac disease: a case report. World J Gastroenterol 2003; 9: 1377-80.

5. Jorge O, Jorge A, Camus G. Celiac disease associated with antiphospholipid syndrome. Rev Esp Enfer Dig 2008; 100: 102-3.

6. Politis EE, Droulias J, Nikolopoulu N, Hadjiconstanti- nou V, apostolou T. Coexisten membranous nephropathy and celiac disease in a 40-year old male. Clin Nephrol 2009; 71: 99-100.

7. Giménez Llort A, Vila Cots J, Camacho Díaz JA, Vila Santandreu A, Concheiro Guisan A, García García L. Nephrotic syndrome associated with celiac disease. A report of five cases. Nephron 2002; 92: 950.

8. Casella G, Perego D, Baldini V, Monti C, Crippa S, Buda CA. A rare association between ulcerative colitis (UC), celiac disease (CD), membranous glomerulonephritis, leg venous thrombosis, and heterozygosity for factor $\mathrm{V}$ Leiden. J Gastroenterol 2002; 37: 761-2.

9. Halma C, Ubels F. Celiac sprue-associated membranous nephropathy. Clin Nephrol 2007; 68: 197.

10. Scholey J, Freeman HJ. Celiac sprue-associated immune complex glomerulonephritis. J Clin Gastroenterol 1986; 8: 181-3.

11. Katz A, Dyck RF, Bear RA. Celiac disease associated with immune complex glomerulonephritis. Clin Nephrol 1979; 11: 39-44.

12. Biyikli NK, Gökçe I, Cakalağoğlu F, Arbak S, Alpay H. The co-existence of membranoproliferative glomerulonephritis type 1 and celiac disease: a case report. Pediatr Nephrol 2009; 24: 1247-50.

13. Ludvigsson JF, Montgomery SM, Olén O, Ekbom A, Ludvigsson J, Fored M. Coeliac disease and risk of renal disease-a general population cohort study. Nephrol Dial Transplant 2006; 21: 1809-15.

14. Pierucci A, Fofi C, Bartoli B, Simonetti BM, Pecci G, Sabbatella L, et al. Antiendomysial antibodies in Berger's disease. Am J Kidney Dis 2002; 39: 1176-82.

15. Smerud HK, Fellström B, Hällgren R, Osagie S, Venge $\mathrm{P}$, Gudjón K. Gluten sensitivity in patients with IgA nephropathy. Nephrol Dial Transplant 2009; 24: 2476-81.

16. Swarbrick ET, Fairclough PD, Campbell PJ, Levison DA, Greenwood RH, Baker LR. Coeliac disease, chronic active hepatitis and mesangiocapillary glomerulonephritis in the same patient. Lancet 1980; 2: 1804-5.

17. Ots M, Uibo O, Metsküla K, Uibo R, Salupere V. IgAantigliadin antibodies in patients with IgA nephropathy: the secondary phenomen? Am J Nephrol 1999; 19: 4538.

18. Fornasieri A, Sinico RA, Maldifassi P, Bernasconi P, Vegni M, D’Amico G. IgA-antigliadin antibodies in IgA mesangial nephropathy (Berger's disease). Br Med J (Clin Res Ed) 1987; 295: 78-80.

19. Collin P, Syrjanen J, Partanen J, Pasternack A, Kaukinen $\mathrm{K}$, Mustonen J. Celiac disease and HLA DQ in patients with IgA nephropathy. Am J Gastroenterol 2002; 97: 2572-6.

20. Sategna-Guidetti C, Ferfoglia G, Bruno M, Pulitano R, 
Roccatello D, Amore A, et al. Do IgA antigliadin and IgA antiendomysium antibodies show there is latent celiac disease in primary IgA nephropathy? Gut 1992; 33: 476-8

21. Rostoker G, Delchier JC, Chaumette MT. Increased intestinal intra-epithelial $\mathrm{T}$ lymphocytes in primary glomerulonephritis. A role of oral tolerance breakdown in the pathophysiology of human primary glomerulonephritides? Nephol Dial Transplant 2001; 16: 513-7.

22. Rennke HG. Secondary membranoproliferative glomerulonephritis. Kidney Int 1995; 47: 643-56.

23. Picchi A, Pasqualini P, D'Aiello I, Cortese B, Micheli A, Limbruno U. Acute ST-elevation myocardial infarction in a 15-year-old boy with celiac disease and multifactorial thrombotic risk. Thromb Haemost 2008; 99: 1116-8.

24. C.O.C Bellamy. Microangiopathies and malignant vascular injury in the kidney. Current Diagnostic Pathology 2004; 10: 36-51.

25. Keir L, Coward RJM. Advances in our understanding of the pathogenesis of glomerular thrombotic microangio- pathy. Pediatr Nephrol 2011: 26: 523-33.

26. Sethi S, Fervenza FC. Membranoproliferative glomerulonephritis: pathogenetic heterogeneity and proposal for a new classification. Semin Nephrol 2011; 31: 341-8.

27. Karoui S, Sellami MK, Laatar AB, Zitouni M, Matri S, Laadhar L, et al. Prevalence of anticardiolipin and antibeta2-glycoprotein I antibodies in celiac disease. Dig Dis Sci 2007; 52: 1096-100.

28. Eremina V, Baelde HJ, Quaggin SE. Role of the VEGFA signaling pathway in the glomerulus: evidence for crosstalk between components of the glomerular filtration barrier. Nephron Physiol 2007; 106: 32-7.

29. Izzedine H, Massard C, Spano JP, Goldwasser F, Khayat D, Soria JC. VEGF signaling inhibition-induced proteinuria: mechanisms, significance and management. Eur J Cancer 2010; 46: 439-48.

30. Eremina V, Jefferson JA, Kowalewska J, Hochster H, Hass M, Weisstuch J, et al. VEGF inhibition and renal thrombotic microangiopathy. N Engl J Med 2008; 358: 1129-36. 